\title{
Se presume culpable: procesos judiciales de ruptura en la literatura Argentina del siglo XIXI
}

\section{Daniela Dorfman}

Daniela Dorfman es Licenciada en Letras por la Universidad de Buenos Aires. En 2007 publicó "El tiempo en Faulkner: la permanencia de lo fugaz." En Estados Unidos y su cultura: Una mirada retrospectiva, editado por Rolando Costa Picazo y Armando Capalbo. Contacto: dorfmanı@bu.edu 
palabras clave

Estado, violencia, ley, ruptura, derecho.

keywords

State, violence, law, rupture, authority.

\section{resumen}

El carácter represivo del sistema judicial sugiere la idea de una imposición ineludible. Esta visión es rebatida por el concepto de "estrategia de ruptura" con que Jacques Vergés postula la posibilidad de que el acusado no acate esa imposición por no reconocer el orden en que se funda ni la autoridad que la infiere. Este trabajo estudia los procesos judiciales representados en la literatura argentina del siglo XIX - cuando el sistema jurídico estaba aún en formación - como escenas de ruptura en que el acusado invierte la acusación y la dirige a sus jueces, representantes de un orden cuyos valores cuestiona. Martin Fierro, Juan Moreira y el unitario de El matadero, todos enfrentan a la justicia y sus mecanismos. Leo aquí ese enfrentamiento, a partir de las consideraciones benjaminianas sobre una violencia conservadora y una fundadora, como proceso dialéctico en que el derecho necesariamente se constituye. Tengo en cuenta asimismo las reflexiones de Derrida sobre el fundamento de la autoridad y las consecuencias del "olvido" de la conformación histórico-política de la concepción de lo ilícito al examinar los procesos judiciales de estos textos como arena de lucha en que dos órdenes se disputan la imposición de nombres, valores y derechos divergentes.

abstract:

The repressive nature of the judicial system suggests that its imposition is inescapable. Jacques Vergés' "rupture strategy" refutes this idea by proposing that the person who is being accused does not necessarily have to tolerate the imposition if he or she does not recognize the legitimacy of the authority imposing it. This study analyzes the prosecutions represented in Argentinean literature of the 19th Century as rupture scenes, in which the prosecuted reverses the accusation towards the authority and the values it embodies. Martin Fierro, Juan Moreira and "el unitario" from El matadero, all confront 
SE PRESUME CULPABLE: PROCESOS JUDICIALES DE RUPTURA EN LA LITERATURA ARGENTINA DEL SIGLO XIX DANIELA DORFMAN

the law and its mechanisms. I study that confrontation as a dialectic process in which the law is constituted by the interaction of what Benjamin called "conservative violence" and "foundational violence". In addition, I will focus on these scenes as the arena where two systems contest the imposition of names, values and rights. In order to do so I will take into consideration Derrida's reflections on the foundations of authority as well as the consequences of the concealment of the historical and political construction of what is considered to be unlawful. 
EN SU DEFENSA DE LA ANTICOLONiALISTA Djamila Bouhired - militante del FLN para liberar Argelia de la dominación francesa - Jacques Vergés recurre a una "estrategia de ruptura", en virtud de la cual posibilita el siguiente intercambio:

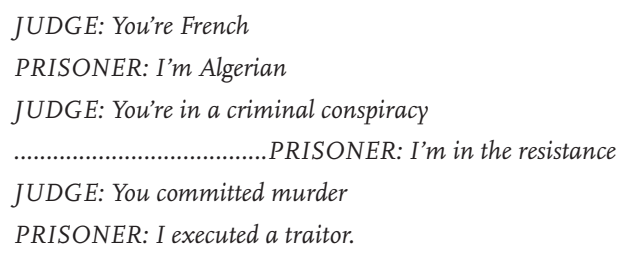

(Terror's Advoca741te) 01

Jacques Vergés exhibe, así, la imposibilidad de todo diálogo entre los participantes, el enfrentamiento por el modo de nombrar - y ya sabemos que nombrar es valorar - un mismo hecho. Cuando esto sucede en un proceso judicial, éste pierde su habilidad de regular las contradicciones entre el individuo y la sociedad porque para ello necesita de la aceptación del acusado. Si éste acepta el orden público, el proceso constituye un diálogo en que el juez - cuyos valores son respetados - intenta determinar la responsabilidad del acusado en un hecho que todos coinciden en considerar delictivo. Estos juicios son "de connivencia” (Vergés, 2008) y suponen una serie de acuerdos - la validez del tribunal, de las leyes y de los procedimientos, el sometimiento al veredicto - y un desacuerdo: la inocencia o culpabilidad del imputado (en algunos casos, la realidad del hecho mismo). Pero si el acusado rechaza el orden público, no reconoce el sistema que lo juzga, el aparato judicial se desintegra; entonces el juicio es "de ruptura" e implica acuerdo acerca de la realidad del hecho y de la culpabilidad del acusado, y desacuerdo en todo lo demás (que el hecho constituya un crimen que deba ser castigado, la legitimidad del tribunal, que el juicio mismo sea una instancia válida). 
Las escenas judiciales ${ }^{\mathrm{I}}$ en la literatura argentina del siglo XIX constituyen una profundización del conflicto porque escenifican la imposibilidad de todo diálogo entre los dos órdenes que allí se enfrentan, lo que resulta en una ruptura por parte del acusado.

En la escena en que el unitario de El matadero es llevado a la casilla del juez e interrogado, estos mantienen un intercambio análogo al referido por Vergés:

JUEZ: ¿Por qué no traes divisa?²

UNITARIO: Porque no quiero

JUEZ: ¿No sabes que lo manda el Restaurador?

UNITARIO: La librea es para vosotros, esclavos, no para los hombres libres

JUEZ: A los libres se les hace llevar a la fuerza

JUEZ: ¿Por qué no llevas luto en el sombrero por la heroína?3

UNITARIO: Porque lo llevo en el corazón por la patria, por la patria que vosotros habéis asesinado, infames

JUEZ: ¿No sabes que así lo dispuso el Restaurador?

UNITARIO: Lo dispusisteis vosotros, esclavos, para lisonjear el orgullo de vuestro señor, y tributarle vasallaje infame. (Echeverría, I999, II6).

Tal como vimos arriba, también aquí se están disputando dos versiones de un mismo hecho, y también aquí esta disputa toma la forma de una diferencia

I Llamo "escenas judiciales" a los episodios en que, dado un enfrentamiento, una de las partes representa la ley o actúa en su nombre. Las considero escenas judiciales aunque no constituyan juicios oficiales, en primer lugar, porque ésta es una de las razones de ruptura por parte de los acusados y, en segundo lugar, porque se pretenden -dentro del sistema que las sostiene - instancias judiciales, si no legítimas, al menos válidas.

2 Divisa punzó, símbolo de lealtad a los federales.

3 Encarnación Ezcurra, difunta esposa de Rosas. 
terminológica (divisa/librea) que alerta contra la pretensión de objetividad con que la justicia impone sus nombres.

En este "proceso" - a diferencia de lo que veremos en los otros textos - se llevan a cabo ciertos actos rituales que presumen oficialidad: dentro del matadero hay una casilla que representa el lugar de la ley; allí hay una mesa, elementos para escribir, un vaso de agua para el acusado, un guardia, un juez; éste sigue algunos procedimientos como hacer poner de pie a los presentes, el interrogatorio, la posibilidad del acusado de defenderse y, finalmente, tras la muerte del unitario, el dar parte. La pretensión de oficialidad y legalidad que estos elementos confieren a la escena realza la drasticidad con que el unitario rechaza todas estas instancias clausurando toda posibilidad de negociación o acuerdo.

La ruptura con el sistema que juzga al unitario viene aquí dada a priori, desde la pertenencia social y - al menos en el texto, consecuentemente - política. Así, las acusaciones en forma de pregunta del juez no se refieren a un hecho o acción por parte del unitario sino a su filiación política, y las respuestas de éste la confirman con determinación. Cada respuesta del unitario subraya la diferencia en que reside su "delito", y cada acción del juez tiene el propósito de corregir esa diferencia para incorporarlo al régimen federal. Por eso - en una escena que sintetiza toda una concepción de los mecanismos con que opera el sistema judicial - el juez manda cortarle la barba en forma de U que identifica a los unitarios y, ante la exhibición provocativa con que el unitario recurre al vocabulario refinado y literario que lo distancia del lenguaje soez y material de los federales, amenaza con cortarle la lengua también. Finalmente, la diferencia que resulta decisiva es la que lleva a la muerte del unitario y que el propio juez formula - ante la imposibilidad de corregirla - diciendo que querían "únicamente divertir[se] con él y tomó la cosa demasiado a lo serio" (Echeverría, I999, I7). Este desacuerdo constituye una manifestación especialmente significativa, por la profundidad que evidencia, de la irreconciabilidad extrema de 
las dos partes enfrentadas. Si el juicio a Djamila Bouhired remitía, al comienzo del trabajo, a la imposibilidad de todo diálogo entre el unitario y el juez de $E l$ matadero, la discordia entre estos sobre lo que es risible nos devuelve, ahora, a ese juicio, en que Djamila ríe al escuchar su sentencia a muerte, exasperando al juez que no puede sino considerarlo un asunto muy serio.

En El matadero, el juez busca anular la diferencia mientras el unitario la exalta. Responde sistemáticamente a sus preguntas y demandas con invectivas que muestran una delectación en la diferencia de que se lo acusa y que ponen en primer plano su pertenencia a un orden otro. No se defiende porque no busca la absolución sino sacar a la luz sus ideas, renuncia a la propia defensa en pos de la exhibición de una causa. ${ }^{4}$ Esta exhibición de la diferencia constituye un acto político que se resiste a ser subsumido en la categoría de "crimen común”. La posibilidad misma de un “crimen de derecho común” es problemática desde el momento en que el Estado se siente afectado y toma parte por considerar que la infracción daña no solamente a la víctima sino al orden todo - al Estado, la sociedad, la soberanía -. En tanto toda infracción es considerada una amenaza al orden - por eso interviene el Estado - todo proceso entraña, entonces, un enfrentamiento político: el hambre, el paro o la prostitución, expresan rupturas profundas que son políticas, por mucho que pertenezcan al ámbito del "derecho común" (Vergés, 2008, 23). La existencia de una categoría tal y de estilos de proceso específicos para este tipo de delitos, constituye un triunfo del derecho - y, por su medio, del Estado - que logra así ocultar bajo la apariencia de un problema moral la expresión de un conflicto que es social. Sin embargo, cuando el acusado presume de su culpabilidad el gesto político se hace difícil 
de encubrir. Esto es lo que hace Fidel Castro cuando responde a las acusaciones de los jueces de Batista con un programa que detallaba no sólo el plan de insurrección sino las primeras leyes que promulgaría una vez en el poder, incluyendo la reforma agraria; o Sócrates en cuya "defensa" insiste en las mismas ideas por las que está siendo acusado de corrupción y herejía, mostrando el carácter premeditado de sus actos en lugar de negarlos (Vergés, 2008, 64). Si el acusado negara o explicara su delito alegando circunstancias excepcionales, estaría reconociendo la existencia y validez - y adoptando para sí los principios - del orden ético que lo juzga; la declaración de culpabilidad, en cambio, es en sí misma la fundación de otra ética, la postulación de otro orden, el rechazo y recusación del vigente. El unitario de El matadero exhibe con orgullo su "delito" y se complace en la impugnación minuciosa del orden ético-jurídico que se le quiere aplicar. Responde a una acusación - más o menos abiertamente - política con ideas políticas disidentes, lo que equivale a aceptar culpabilidad y hace trizas el orden que lo juzga porque lo desconoce, y erige un orden paralelo al cual adscribe. Por eso ostenta el unitario - ya veremos que también Juan Moreira - su culpabilidad, subrayando su no pertenencia a ese orden.

Aquí es justamente donde se diferencia el Martín Fierro. Éste, tras ser maltratado en la frontera que debe defender de los indios como servicio a su país, deserta y regresa a su rancho. Al llegar no encuentra sus cosas, mujer ni hijos, jura “en esa ocasión/ ser más malo que una fiera” (Hernández, I960, 46) y pasa dos años como "gaucho matrero". En virtud de la ley que sanciona la falta de residencia fija de los gauchos - carencia generada por el propio sistema, que los envía a la frontera y se adueña de sus propiedades - la justicia persigue a Martín Fierro. Éste la enfrenta tan valientemente que uno de los soldados de la partida se pasa a su bando; juntos derrotan a la policía y deciden irse con los indios al otro lado de la frontera, donde “no alcanza la facultá del gobierno” (Hernández, I960, 8I). Antes de irse, Martín Fierro realiza esa ruptura en el cuerpo 
de la guitarra con que había acompañado el relato de su vida. Serán necesarios siete años - cinco en el tiempo de la ficción - para que Fierro vuelva a empuñar la guitarra y retorne a la civilización. Vuelve, entonces, al pago y aunque nadie recuerda ya sus crímenes él mismo los somete a un auto-enjuiciamiento en el que, a diferencia de lo que sucede cuando hay ruptura, trata de justificarse.

La partida de Martín Fierro es problemática en tanto ruptura justamente por su radicalidad, dado que al ser tan extrema se vuelve invisible a las autoridades. Al abandonar la sociedad que lo maltrata está sencillamente “anulando" el problema puesto que las autoridades ya no tienen que enfrentar su disidencia. Pero ¿sería, una ruptura menos extrema, expresión de una reprobación mayor? Probablemente no. Sin embargo, la aceptación de la autoridad que el comparecer ante ella inicialmente supondría es necesaria para realizar y hacer efectivo el desacuerdo ulterior. Para realizar la ruptura el acusado debe comparecer ante la justicia para, al no dar testimonio, dar testimonio de su oposición al orden que le reclama que comparezca. Así es que, aunque en apariencia mengüe la radicalidad de la ruptura, es necesario que el acusado se presente ante la justicia y haga, allí, expresa su protesta. Por eso la ruptura de Martín Fierro - por radical - no es efectiva en tanto rechazo del orden, dado que no lo hace manifiesto, es una especie de rechazo in absentia, que no llega por eso a constituirse en acusación.

La ruptura total y la posterior reinserción a la ley y a la sociedad de Martín Fierro es sólo una de las posibilidades tras la confrontación con la partida de policías; otra es la que realiza Juan Moreira (Ludmer, I999). Cuando, en un principio, el alcalde acosa a Moreira con citaciones, multas y cepos éste lo acepta, se somete a la justicia y consiente, renuente, sus castigos. En este primer momento los procesos judiciales son de connivencia. Moreira no está de acuerdo en su culpabilidad pero respeta el orden establecido y participa de sus instancias legales. Acude, entonces, a esa misma justicia para cobrar una 
deuda y aquí se produce el choque de dos códigos: el pacto oral hecho entre Sardetti y Moreira no tiene validez en el código escrito de la justicia oficial. Sardetti niega la deuda, el alcalde acusa de mentiroso a Moreira y entonces se produce la ruptura y Moreira comienza a regirse por otra legalidad. Visita, entonces, a Sardetti y le cobra la deuda estableciendo su propio sistema de equivalencias una puñalada por cada mil pesos - ateniéndose así a una justicia paralela que, en tanto tal, cuestiona la vigente. Este ajusticiamiento lo pone fuera de la ley y da comienzo a su persecución. Durante el resto de su vida, y de la novela que termina con ella, es buscado por la justicia que finalmente lo alcanza y lo deja clavado de un pulmón a la pared.

A diferencia de Martín Fierro, Juan Moreira procura manifestar reiteradamente su ruptura. Aquél, perseguido, cruza la frontera; éste, en cambio, se va del pueblo pero vuelve para matar al alcalde, no sin antes explicarle porqué; enfrenta en lucha armada a las partidas; tras matar a Leguizamón va al juzgado y pide al sargento que le alcance la daga, que había “olvidado” prolijamente en el cuerpo del muerto; cuando se entera de que el juez de paz de Cañuelas está reforzando su partida con soldados de otros pueblos para perseguirlo, va él mismo a la puerta del juzgado y anuncia que él es Juan Moreira y que viene a pelearlos. Semejante audacia es toda una afirmación que se hace explícita cuando al ganar la pelea perdona la vida al capitán pero le ordena ir a llevar el parte. Después de esto todavía tres veces más - una en Matanzas, otra en Navarro y la última en Salto - va él al juzgado, golpea la puerta y se anuncia a gritos a las partidas que dicen buscarlo pero no le abren. Al final, será la justicia quien golpee a su puerta en "La estrella” y él sí abrirá y los enfrentará una última vez. La ruptura de Juan Moreira es menos radical que la de Martín Fierro pero su denuncia resulta más incisiva en virtud de la confrontación sostenida y la postulación de un orden alternativo - lo cual posiblemente le haya costado la designación del Martín Fierro como texto nacional -. 
Las rupturas que estos textos presentan frente a la institución jurídica y judicial son motivadas por leyes y coyunturas particulares como el usufructo del cuerpo del gaucho por el Estado, abusos de autoridad, la ley de leva, la aplicación desigual de la ley, apropiaciones ilegítimas de las propiedades del gaucho, persecuciones, etc. Pero en su impugnación total del orden ponen en evidencia el carácter político de todo proceso y develan una serie de convenientes "olvidos" que permiten que la ley se presente como algo dado, universal y ahistórico, como la voz de la moral y de la convivencia pacífica. Esos "olvidos" operan en función de uno mayor: el que oculta la coimplicancia entre ley y violencia y la pertenencia del concepto de violencia al campo simbólico del derecho mismo. La palabra alemana Gewalt que Benjamin analiza en su Zur Kritik der Gewalt y que significa al mismo tiempo "violencia” y "fuerza de ley", “violencia autorizada” y “poder legal”, repone esa relación que el derecho quiere hacernos olvidar. Esta violencia legal, autorizada en tanto institucionalizada por el Estado, tiene la función de conservar el orden de cosas, para lo cual debe reprimir "contraviolencias hostiles" (Derrida, I997, I33). Para lograrlo, el derecho se establece a sí mismo declarando violento y, por tanto, fuera de la ley todo aquello que no lo reconoce. Mediante esa formulación tautológica, toda violencia que apunte a fundar otro derecho está, por definición, fuera de la ley. El derecho rehúsa así al sujeto individual el uso de la violencia y utiliza los aparatos estatales para impedir que otras violencias se presenten como teniendo derecho al derecho (Derrida, I997).

Por eso, y en tanto su ruptura se expresa por medio de una violencia no autorizada, Martín Fierro y Juan Moreira plantean el problema de la legitimidad de la violencia a la que recurren. Cuando un acusado disputa el monopolio de la violencia al Estado y adopta la estrategia de ruptura, lo hace en nombre de un orden que no está vigente aún pero que legitimará su violencia retroactivamente cuando lo esté (Derrida, I997). Este desfase pone al descubierto el devenir 
de la "violencia fundadora" en "violencia conservadora" al fundarse el nuevo orden. Es por eso que la violencia que busca fundar un nuevo orden de cosas puede herir nuestro sentimiento de justicia e incomodarnos cuando es leída, desde una concepción de violencia y crimen, solidaria con la que está tratando de rebatir. Esto supone la conformación histórica no sólo de la ley sino de aquello que se concibe como delito en un momento dado, y si la ley es forjada históricamente, y está amenazada por otros órdenes y derechos está, entonces, en permanente desarrollo, por lo que habría un remanente de indecidibilidad en virtud del cual no es nunca posible deslindar de modo concluyente lo correcto de lo incorrecto.

Así, al invertir la acusación y recordar la conformación histórica y provisional de la ley, la ruptura no sólo cuestiona el sistema vigente sino que también restituye el carácter político y violento de todo sistema judicial. Al enfrentar la ley, los protagonistas de los textos analizados parecen hacer las mismas preguntas que el comunista húngaro Rakosi hacía a sus magistrados, en I925: “¿Quiénes sois?”, “¿Qué representáis?”, “¿Cuál es vuestra razón histórica de ser?” (Vergés, 2008, 27). Estas preguntas tienen el mérito de reconocer el carácter representativo que cada parte tiene en todo proceso, desenmascarando la pretensión de neutralidad de los tribunales. Permiten leer, además, las fuerzas que se enfrentan allí, así como el proyecto de Estado que cada concepción estaba proponiendo, removiendo la violencia jurídico-estatal del lugar de privilegio a que aspira al arrogarse neutralidad y auto-sancionarse portadora de la Justicia, para presentarla en su verdadero carácter político, transitorio y conservador. En su ruptura, el unitario, Martín Fierro y Juan Moreira se presentan, así, como la contracara del campesino de "Ante la ley" quien, por obedecer la ley que le ordenaba quedarse afuera, muere de viejo ante una puerta que le estaba destinada pero él no acomete, impávido ante el guardián que le dice "intenta entrar a pesar de mi prohibición." (Kafka, I974, 20). 
SE PRESUME CULPABLE: PROCESOS JUdiCIALES DE RUPTURA EN LA LITERATURA ARGENTINA DEL SIGLO XIX DANIELA DORFMAN

\section{REFERENCIAS BIBLIOGRÁFICAS}

Derrida, Jacques, "Nombre de pila de Benjamin”. Fuerza de ley. El "fundamento místico de la autoridad”. Madrid: Editorial Tecnos, I997.

"Kafka: Ante la ley". La filosofía como institución. Barcelona: Juan Granica, 1984 .

Echeverría, Esteban, El matadero. Buenos Aires: Talcas Ediciones, I999.

Gutiérrez, Eduardo, Juan Moreira. Barcelona: Editorial Sol 9०, 200 I.

Hernández José, Martín Fierro. Buenos Aires: EUDEBA, I960.

Vergés, Jacques, Estrategia judicial en los procesos políticos. Barcelona: Editorial Anagrama, 2008.

Kafka, Franz, “Ante la ley”. En: Cuentos. Buenos Aires: Ediciones Orión, I974.

Ludmer, Josefina, “Los Moreira”. Juan Moreira. Buenos Aires: Libros Perfil, I999.

Terror's Advocate. Dir. Barbet Schroeder. Magnolia Pictures, 2007. DVD. 
